\title{
IN-AIR ULTRASONIC RANGEFINDING AND ANGLE ESTIMATION USING AN ARRAY OF ALN MICROMACHINED TRANSDUCERS

\author{
R.J. Przybyla ${ }^{1 *}$, S.E. Shelton ${ }^{2}$, A. Guedes ${ }^{2}$, R. Krigel ${ }^{l}$, D.A. Horsley ${ }^{2}$, and B.E. Boser ${ }^{l}$
} \\ Berkeley Sensor and Actuator Center \\ ${ }^{1}$ University of California, Berkeley, CA, USA \\ ${ }^{2}$ University of California, Davis, CA, USA
}

\begin{abstract}
We describe a 2D ultrasonic depth sensor based an array of piezoelectric micromachined ultrasound transducer elements which measures range and direction to targets in air. The transducer consists of a single transmitter and seven receive elements arranged in a $6 \mathrm{~mm}$ long linear array with half wavelength spacing. The system employs pulse-echo time-of-flight and difference of arrival time to determine target range and angle and operates up to a $750 \mathrm{~mm}$ maximum range and $\pm 35^{\circ}$ angle span with $\pm 3.5 \mathrm{~mm}$ range and $\pm 3^{\circ}$ angle worst case errors, respectively.
\end{abstract}

\section{INTRODUCTION}

Optical solutions presently dominate $2 \mathrm{D}$ and $3 \mathrm{D}$ ranging systems. Although capable of relatively high resolution and frame rate, these systems suffer from high power dissipation typically in excess of several Watts [1], precluding their use in mobile applications for gesture recognition and similar applications. A further drawback of optical solutions is their sensitivity to ambient illumination and limited performance in sunlight.

Ultrasonic transducers overcome these drawbacks. Modern micromachined single element or small transmitter arrays generate sufficient ultrasonic power from a sub- $\mathrm{mW}$ electrical drive signal for target ranges up to a few meters. Compared to light, ultrasonic waves travel at roughly one million times slower velocity, translating into simpler and lower bandwidth electronics that translate into further power savings compared to optical solutions. High frequency ultrasound $(>100 \mathrm{kHz})$ is particularly attractive for chip-scale systems because several transducer elements spaced at quarter wavelength fit across a cm-sized transducer die, thus enabling $2 \mathrm{D}$ and $3 \mathrm{D}$ ranging in a very compact form factor.

Previous ultrasonic depth sensors use bulky piezoceramic arrays [2] or use large transmitters for excitation [3]. In this work, we present a $2 \mathrm{D}$ rangefinder based on a chip-scale batch-fabricated aluminum nitride (AIN) ultrasonic transducer array. We discuss the factors that affect the performance of a depth sensor, and use these to design a system which is suitable for simple gesture recognition.

\section{MICROMACHINED ULTRASOUND TRANSDUCERS}

The ultrasound transducers used in this work [4],[5] are circular piezoelectric unimorph membranes. Figure 1 shows the arrangement consisting of a $2 \mu \mathrm{m}$ thick $\mathrm{AlN} / \mathrm{Mo} / \mathrm{AlN} / \mathrm{Al}$ stack fabricated on a silicon wafer and released with a backside throughsilicon etch. Voltage applied across the electrodes results an inplane stress in the top AlN layer via the inverse piezoelectric effect. The bottom layer of AlN causes a stress gradient to form across the membrane which results in out-of-plane displacement, causing the transducer to emit a pressure wave. Similarly, an incident pressure wave causes in-plane stress in the top layer of AIN, which results in charge developing across the electrodes that can be sensed electrically.

An electrical, mechanical, and acoustical model for the device is presented in [6]. The $450 \mu \mathrm{m}$ diameter transducers are designed to have a resonant frequency $f_{o} \approx 190 \mathrm{kHz}$. At atmospheric pressure, the $\mathrm{Q}$ of the transducer is about 15 , corresponding to a bandwidth $B W=f_{d} / 2 Q \approx 6.2 \mathrm{kHz}$, and the motional impedance of each device is

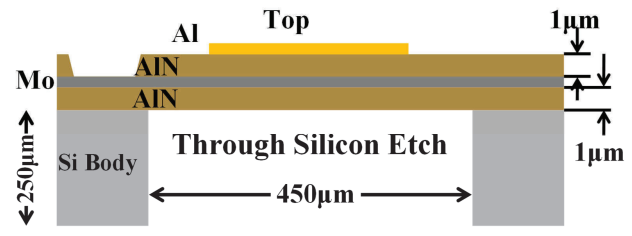

Figure 1: Schematic cross-section of a single pMUT.

Table 1: Depth Sensor Design Specifications

\begin{tabular}{l|l} 
Specification & Value \\
\hline \hline Max Range & $500 \mathrm{~mm}$ \\
Angular span & $60^{\circ}$ \\
Range accuracy & $10 \mathrm{~mm}$ \\
Range Resolution & $50 \mathrm{~mm}$ \\
Angular Accuracy & $2^{\circ}$ \\
Angular Resolution & $15^{\circ}$ \\
Update rate & $>30 \mathrm{~Hz}$ \\
\hline
\end{tabular}

approximately $2 \mathrm{M} \Omega$ at resonance. The effective surface area of each transducer is $\mathrm{SA}=0.05 \mathrm{~mm}^{2}$. The parallel plate capacitance of these devices, including bond pads and interconnect, is approximately $8 \mathrm{pF}$.

\section{SYSTEM DESIGN}

Table 1 lists the target specifications for a 2D ultrasonic depth sensor that is suitable for simple user interface tasks such as advancing a slide. Below we discuss the design of the measurement system for range and angle measurements as well as error sources that limit the range and accuracy of the system.

\section{Range Measurement}

The ranger operates by emitting an ultrasound signal and measuring the echo from a target. The transmitted signal can be either a continuous wave or a pulse. Continuous time solutions are attractive for narrow-band transmitters but suffer from multi-path fading and high receiver dynamic range requirements due to feedthrough from the transmitter to the receiver. For this reason, a pulse-echo design is used in this implementation. In this case, a short pulse is transmitted and the range $R$ estimated from the speed of sound $c \approx 340 \mathrm{~m} / \mathrm{s}$ and the delay $T=2 R / c$ of the echo. For a target at $R=1 \mathrm{~m}$, the delay is approximately $5.9 \mathrm{~ms}$.

An ideal system would transmit a narrow, sharp pulse. Short duration permits resolving closely spaced targets, while short rise time is critical to minimize timing errors due to amplitude fluctuations. Consequently, in practical, band limited systems, it is desirable to use all of the available bandwidth. In practice, this means the bandwidth of the system should be designed to be limited by the bandwidth of the ultrasound transducer, and not the interface electronics.

The ideal transmit signal is approximated in practice with a burst of a sinusoidal signal at the resonant frequency of the transmitter element. The duration $T_{p}$ of the burst involves a tradeoff between minimum and maximum target range. Since the receiver is disabled during transmission, the minimum target 
distance is $R_{\text {min }}=c T_{p} / 2$. However, bursts much shorter than $1 /(2 \mathrm{BW}) \approx 80 \mu \mathrm{s}$ corresponding to $R_{\text {min }} \approx 14 \mathrm{~mm}$ result in reduced transmit amplitude and hence smaller maximum range.

Targets spaced less than $\sim c T_{p} / 2$ in range result in overlapping echoes which are difficult to resolve as separate targets [7]. The pulse also broadens as it passes through the narrowband transducer. For a pulse that is shaped by a second order transducer response on transmission and reception, the range resolution is:

$$
\Delta_{r}=\frac{c}{2}\left(\mathrm{~T}_{\mathrm{p}}+\frac{0.27}{\mathrm{BW}}\right)
$$

For the numbers used above, the range resolution is $\Delta_{r} \approx 21 \mathrm{~mm}$.

The maximum useful range is limited by the maximum output pressure, thermal noise from the air, and noise from the electronic pickup circuit. The transmit pressure of a single element at $f_{o}$ is limited to $p_{t x} \approx 500 \mathrm{~Pa}$ by the third-order Duffing nonlinearity of the clamped-clamped membrane. Depending on the design of the transducer, the maximum output pressure will either be limited by the maximum available transmit voltage or mechanical nonlinearity.

As the ultrasonic pulse travels through the air, it is attenuated due to spreading and absorption. The roundtrip acoustic path loss is given by [8]:

$$
G_{p}=G_{a c} \frac{R_{o}}{4 R} 10^{-2 \alpha R}
$$

In this equation, $R$ is the range to the target. $\alpha$ is the attenuation constant, which is strongly dependent on frequency, and also dependent on humidity and ambient pressure [8]. At $190 \mathrm{kHz}, \alpha$ $\approx 10 \mathrm{~dB} / \mathrm{m}$. $G_{a c}$ is the acoustic gain, which depends on the size of the target, and which can also be increased by using a horn. For large targets in this design, $\mathrm{G}_{\mathrm{ac}}=20 . R_{o}=\mathrm{SA} / \lambda=27 \mu \mathrm{m}$ is the Rayleigh range, and $\lambda=c / f_{o}=1.8 \mathrm{~mm}$ is the wavelength.

The signal received by the transducer at $f_{o}$ is $i_{r x}=$ $\left(p_{r x} / \mathrm{R}_{\mathrm{m}}\right)(S A / \eta)=8 \mathrm{nA} / \mathrm{Pa}$, where $\mathrm{R}_{\mathrm{m}}$ is the motional resistance of the transducer. Random air molecule thermal vibrations are the dominant source of noise from the transducer. This noise signal is shaped by the transducer dynamics. The electronic amplifier also adds wideband thermal noise which must be filtered electronically for optimal noise performance. As mentioned above, the bandwidth of the system is critical to resolution and accuracy, so the electronic filter should not restrict the overall bandwidth of the system unless additional range is required.

For pulse detection, a threshold is generally used to distinguish faint echoes from noise. The threshold must be set high enough to detect echoes at the maximum range but to reject noise pulses which would otherwise cause large errors in the range estimate. The average time between false alarms is given in [7]:

$$
\mathrm{t}_{\mathrm{fa}}=\frac{1}{\mathrm{BW}} \mathrm{e}^{\mathrm{V}_{\mathrm{TH}}^{2} / \sigma_{\mathrm{A}}^{2}} \text {. }
$$

In practice, $\mathrm{V}_{\mathrm{TH}}^{2} / \sigma_{\mathrm{A}}^{2}=12 \mathrm{~dB}$ is a good target and corresponds to $\mathrm{t}_{\mathrm{fa}}=24$ minutes, an acceptable value in most applications. Increasing the threshold reduces the frequency of false alarms at the expense of the theoretical maximum range.

Since the dominant noise sources in the system are in the receiver, increasing the number of receive elements increases the signal power by $\mathrm{N}^{2}$ but also increases the noise power by $\mathrm{N}$. Adding transmit elements does not appreciably increase the total noise. Consequently, the on-axis SNR for a system with $\mathrm{N}_{\mathrm{tx}}$ transmitters and $\mathrm{N}_{\mathrm{rx}}$ receivers is [9]:

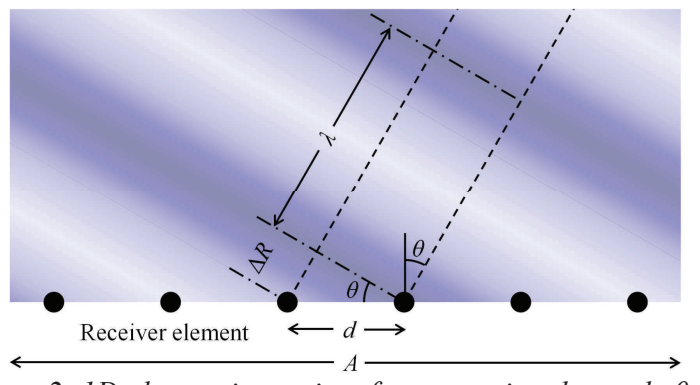

Figure 2: 1D ultrasonic receiver for measuring the angle $\theta$ of the target based on the difference in arrival time $\Delta T=\Delta R / c$ between adjacent elements.

$$
\mathrm{SNR}=\mathrm{N}_{\mathrm{tx}}^{2} \mathrm{~N}_{\mathrm{rx}} \frac{\left(\frac{\mathrm{SA}}{\eta} \frac{1}{\mathrm{R}_{\mathrm{m}}}\right)^{2} p_{t x}^{2} G_{p}^{2}}{\frac{\mathrm{kT}}{L}+\bar{v}_{a}^{2}\left(\omega_{o} C_{o}\right)^{2} \mathrm{NBW}},
$$

where $\mathrm{kT}$ is the thermal energy of the system, $L=25 \mathrm{H}$ is the effective motional inductance of the transducer, $\bar{v}_{a}^{2}$ is the spot noise variance of the front-end amplifier, $\omega_{o}=2 \pi f_{o}, C_{o}=8 \mathrm{pF}$ is the parasitic capacitance of the transducer, and NBW is the effective noise bandwidth of the electronic filter. The LHS of the denominator is mechanical noise from the transducer, and the RHS is electronic noise from the front-end amplifier. Since the noise does not depend on range, equations (2) and (4) show that the signal decreases linearly with increased range at relatively short ranges, and then begins to decrease much faster as absorption starts to become a dominant loss factor. With our system, the theoretical maximum range is $800 \mathrm{~mm}$ for a large target, and at $500 \mathrm{~mm}$ distance the SNR is $25 \mathrm{~dB}$ [9].

Amplitude noise $\sigma_{a}$ in the received signal, combined with finite burst rise time, translates into an equivalent range noise [7]:

$$
\sigma_{r}=\frac{c}{2 \mathrm{BW}} \frac{1}{2.1(\mathrm{SNR})^{1 / 2}}
$$

At the maximum range, $\mathrm{SNR}=12 \mathrm{~dB}$, which results in a worst-case $\sigma_{r}=3.3 \mathrm{~mm}$.

\section{Angle Measurement}

The goal of an angle measurement system is to measure the direction to the target from the transceiver. Figure 2 depicts a linear array of receivers used to receive a plane wave returning from a target. An incident angle $\theta$ creates a difference in the timeof-flight between neighboring elements $\Delta T=d / c \sin (\theta)$ which corresponds to a phase shift $\phi=2 \pi d / \lambda \sin (\theta)$, where $\lambda$ is the wavelength of the sound wave. Similarly, to transmit a pulse with angle $\theta$ from normal, the phase of the transmit signal should be shifted by $\phi$ between each element.

As shown in equation (4), an N-element receive array will only increase the SNR by a factor of $\mathrm{N}$ compared to a single transceiver. However, since the receive signals can be summed in the electrical domain, the signals can be shifted so that the phases align irrespective of the input phase difference. Because of this flexibility, a receive array can maintain a constant SNR across a wide span of angles.

For a linear array of $\mathrm{N}$ transducers spaced distance $d$ apart, we define the aperture of the system as $A=\mathrm{N} d / \lambda$. Aperture in the angle axis is analogous to bandwidth in the range axis, so increasing the effective aperture leads to increased angle measurement precision.

The aperture of the system determines the angular beam width of both the transmitted signal and the received signal. The 


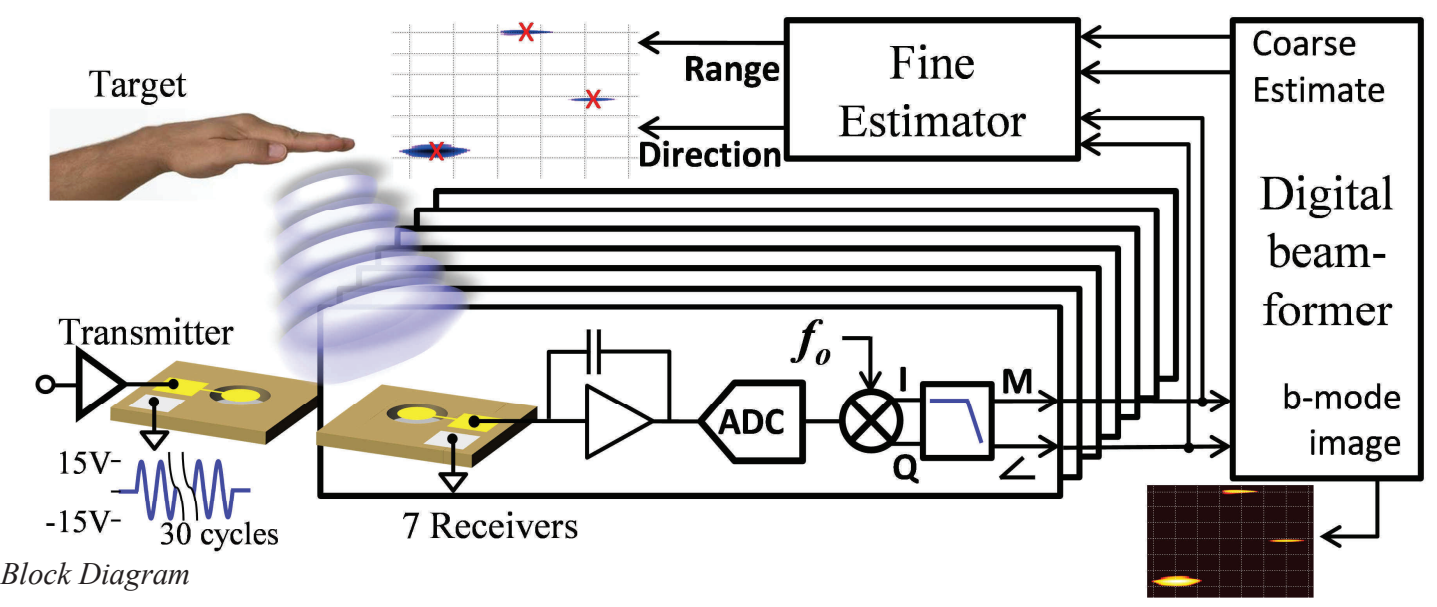

Figure 3: Block Diagram

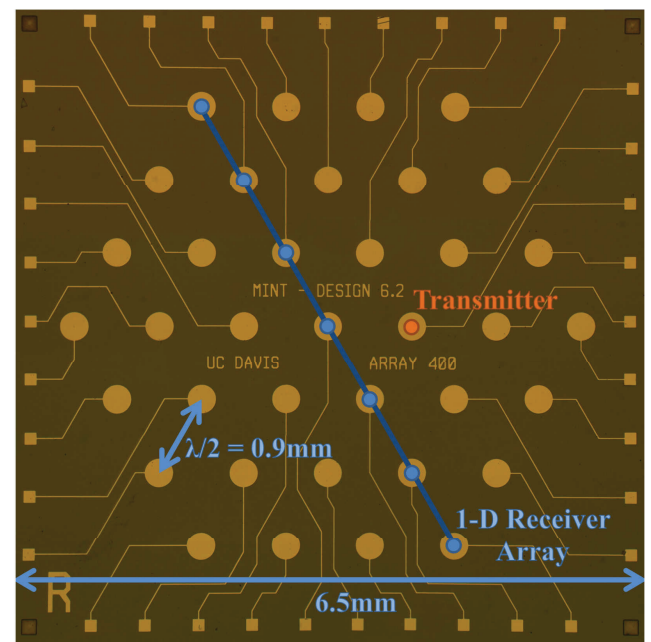

Figure 4: Optical image of a hexagonal pMUT array showing single transmit device and linear sub-array of receive devices.

directivity as a function of angle $\theta$ for a line array of isotropic sources can be shown [8] to be:

$$
D(\theta)=\frac{\sin \left(\mathrm{N} \frac{\pi d}{\lambda} \sin \theta\right)}{\mathrm{N} \sin \left(\frac{\pi d}{\lambda} \sin \theta\right)},
$$

where $d$ is the spacing between elements. To avoid large sidelobes in the directivity response, $d$ should be $\leq \lambda / 2$. We set $d=\lambda / 2$ in this design, and use a 7 element receive array, hence $A=3.5$.

For $\mathrm{N} \gg>1$, the small-angle approximation can be used to estimate the full-width half-power beam width $\theta_{b}$ as

$$
\theta_{b} \cong \frac{2.78 \lambda}{N \pi d} \cong \frac{0.886}{A} \text {. }
$$

This equation gives the beam width for a single array. If transmit and receive arrays are used, the product of the transmit and receive directivity functions must be solved for $\theta_{b}$. $\theta_{b}$ is the angular resolution of the system; for two distinct targets at the same range to be individually resolvable they must be separated by at least $\theta_{b}$ degrees. With $A=3.5, \theta_{b}=14.5^{\circ}$.

The aperture also affects the angular accuracy of the system. The rms angle error $\sigma_{\theta}$ given in [7] is

$$
\sigma_{\theta} \cong \frac{0.628 \theta_{b}}{(\mathrm{SNR})^{1 / 2}} \cong \frac{0.556}{A(\mathrm{SNR})^{1 / 2}} .
$$

At the maximum range, $\mathrm{SNR}=12 \mathrm{~dB}$, and $\sigma_{\theta}=2.3^{\circ}$, which is greater than the specification. However, at $500 \mathrm{~mm}, \mathrm{SNR}=25 \mathrm{~dB}$, which corresponds to an rms angle error $\sigma_{\theta}=0.5^{\circ}$.

Equations (7) and (8) suggest that the aperture $N d / \lambda$ should be made as large as possible to achieve the best angular performance. However, since $d \leq \lambda / 2$, achieving a smaller angular resolution requires increasing the size of the array or increasing the operating frequency. Since absorption losses in equation (2) increase at higher frequency, there is a tradeoff between angular resolution and maximum range for a given chip size.

\section{IMPLEMENTATION}

Figure 4 shows a micrograph of the seven element linear array used to receive echoes from the environment. To protect the transducer membranes from damage, the backside of the die is exposed to the air. The block diagram in Figure 3 illustrates the operation. The transmitter is excited with a $\mathrm{T}_{\mathrm{p}}=160 \mu \mathrm{sec}$ burst at $f_{o}$ and emits an omnidirectional beam. We use a transmit signal twice as long as required by the bandwidth of the transducer to allow more accurate measurement of the phase of the return echo; this increases the range resolution from $21 \mathrm{~mm}$ to $34 \mathrm{~mm}$. The transmitter power consumption, based on $\mathrm{CV}^{2} f$ losses, is $50 \mu \mathrm{W}$. The 1D receiver array captures the echoes which are amplified, digitized, and quadrature down-converted for each channel separately. Digital filtering removes wideband electronic noise. Object positions are calculated by a real-time digital postprocessor. For each angle $\theta$, the signals from each channel $k$ are shifted by a phase shift $k \phi=k 2 \pi d / \lambda \sin (\theta)$ and summed with the other channels. The angle is swept over the entire angle range, resulting in an image such as the one shown in Figure 7. Approximate target ranges and angles are extracted from this data. Subsequently, a fine estimator improves the estimate by searching each baseband signal for a pulse in temporal proximity to the coarse estimate. Refined estimates for the range and angle to the target are determined from the average time-of-flight for each channel's echo and the phase difference between adjacent receive channels.

\section{EXPERIMENTAL RESULTS}

The maximum range is limited by the signal-to-noise ratio and is dependent on the target size; the maximum range is approximately $750 \mathrm{~mm}$ for a metallic cylindrical target with $63 \mathrm{~mm}$ diameter. At $500 \mathrm{~mm}$, the $\mathrm{SNR}$ is $28 \mathrm{~dB}, 3 \mathrm{~dB}$ higher than the design value of $25 \mathrm{~dB}$. This could be due to a conservative estimate of the output pressure. Feedthrough between the transmitter and the receivers causes a dead-time during which no echo can be received, limiting the minimum range to $170 \mathrm{~mm}$ in this prototype. This is longer than the $50 \mathrm{~mm}$ expected from exponential settling of 


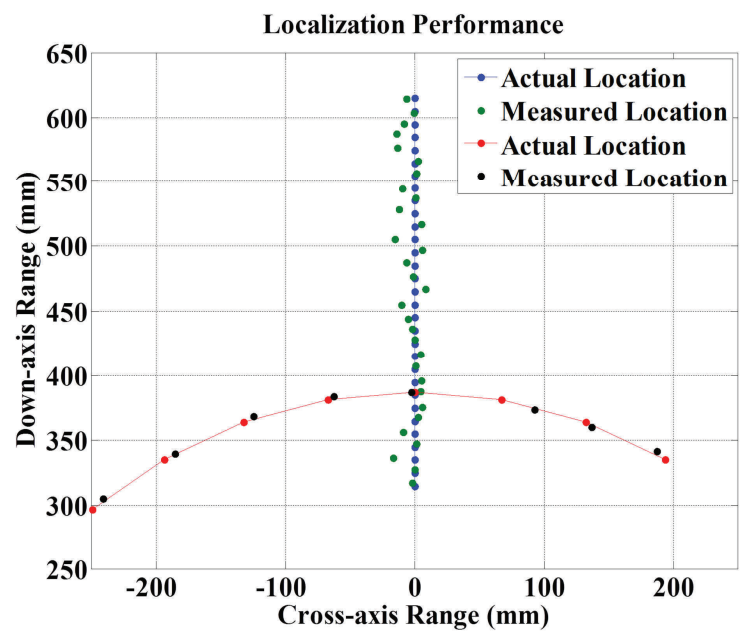

Figure 5: Measurement results showing target localization performance for distance and angle sweeps.

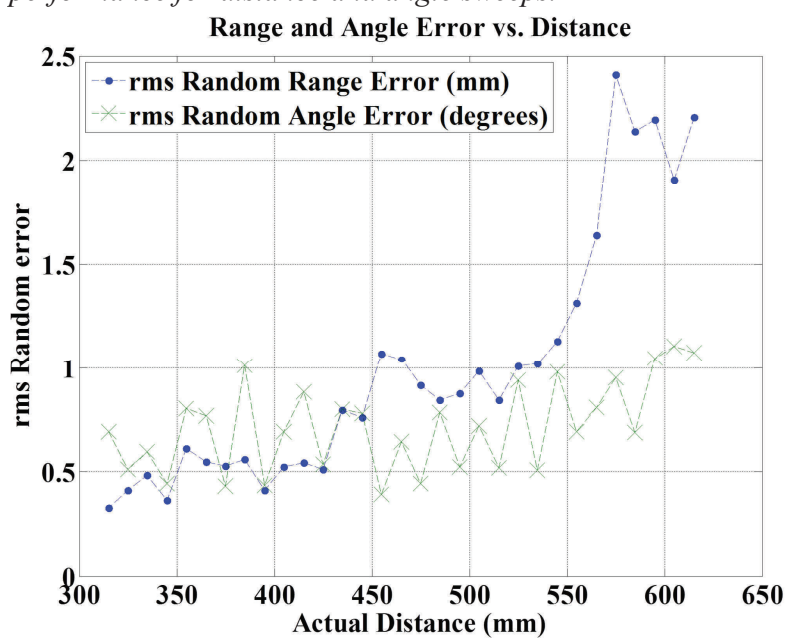

Figure 6: Random range error and random angle error vs. measured distance.

the transmit signal, and may be due to spurious short-range echoes from the test setup. The angular range of the system is $+30^{\circ}$ to $40^{\circ}$. The asymmetry is the consequence of misalignment of the transmitter with respect to the center of the receivers. The measurement time is set by the time-of-flight of the ultrasound pulse and is $4.4 \mathrm{~ms}$ at the maximum range.

Figure 5 shows the range and angle measurement performance with the cylindrical target. The target was moved both radially and on an arc with constant distance from the sensor at location 0,0 (below the plot area), and the plot shows a single representative measurement at each location; no averaging was used. The mean errors were also estimated from 100 recordings at each position and are between $\pm 1.5 \mathrm{~mm}$ and $\pm 2^{\circ}$ for the radial and $\pm 3.5 \mathrm{~mm}$ and $\pm 3^{\circ}$ for arc cases, respectively. Figure 6 shows the random range and angle error versus distance.

Figure 7 demonstrates the system's ability to create a 2D map of an author's head and hands when posing as illustrated.

\section{CONCLUSION}

We have demonstrated the first chip-scale ultrasonic depth sensor, which uses an array of thin-film aluminum nitride transducers to measure range and direction to targets in the field of view. Compared to optical solutions, ultrasonic depth sensors offer

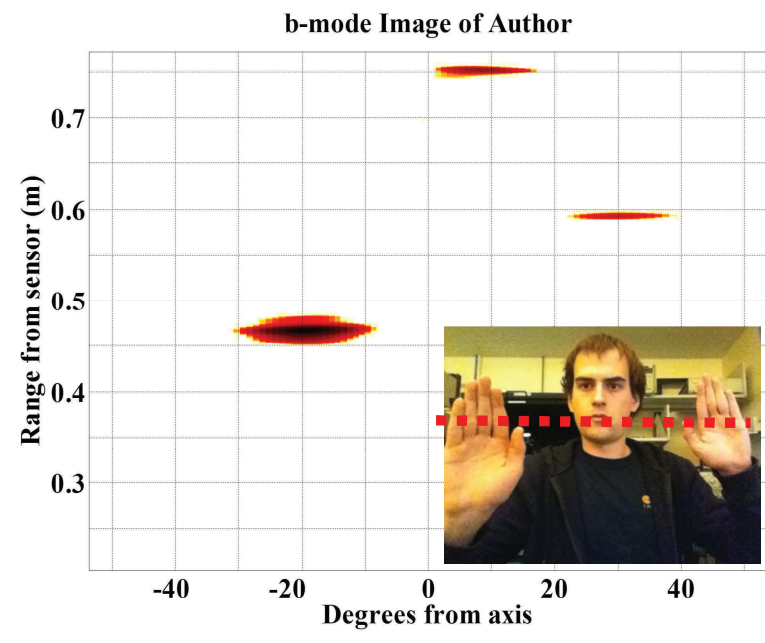

Figure 7: Ultrasound intensity plot showing echo from author's hands and head. Inset: author's pose, showing sensitive axis of system.

orders-of-magnitude reduction in power consumption, ambient light insensitivity, and chip-scale solution size. Combined with good range and angular resolution, ultrasonic solutions are ideal for simple gesture recognition and similar tasks for smart phones and other power constrained devices.

\section{REFERENCES}

[1] Microsoft Corp., Kinect Sensor Manual and Warranty, 2010.

[2] H. Furuhashi et al., "Three-dimensional imaging sensor system using an ultrasonic array sensor and a camera," in Proc. IEEE Sensors 2010, pp. 713-718, 1-4 Nov. 2010.

[3] K. Yamashita et al., "Ultrasonic micro array sensors using piezoelectric thin films and resonant frequency tuning," Sensors and Actuators A: Physical, vol. 114, no.2-3, pp. 147153, Sept. 2004.

[4] S. Shelton, et al., "CMOS-compatible AlN piezoelectric micromachined ultrasonic transducers," in Proc. IEEE Ultrasonics Symp. 2009, Oct. 2009, pp. 402-405.

[5] S. Shelton, et al., "Aluminum nitride piezoelectric micromachined ultrasound transducer arrays," in Proc. Hilton Head Solid-State Sensors, Actuators, and Microsystems Workshop 2012, June 2012.

[6] R. Przybyla, et al., "In-air rangefinding with an AIN piezoelectric micromachined ultrasound transducer," IEEE Sensors Journal, vol.11, no.11, pp.2690-2697, Nov. 2011.

[7] M. Skolnik, Introduction to Radar Systems. $3^{\text {rd }}$ edition, McGraw-Hill, 2001.

[8] D. Blackstock, Fundamentals of Physical Acoustics. John Wiley \& Sons, 2000.

[9] R. Przybyla, et al., "A Micromechanical Ultrasonic Distance Sensor With $>1$ Meter Range," in Proc. Solid-State Sensors, Actuators and Microsystems Conference (TRANSDUCERS) 2011, pp.2070-2073, Beijing, China, 5-9 June 2011.

\section{ACKNOWLEDGEMENT}

This material is based upon work supported by the Defense Advanced Research Projects Agency (DARPA) and/or the Space and Naval Warfare Center, San Diego (SPAWAR SSC-SD) under Contract No. N66001-08-C-2023.

\section{CONTACT}

*Richard J. Przybyla, rjp@berkeley.edu. 